\title{
COMMENTARY
}

\section{A protocol guided by transpulmonary thermodilution and lactate levels for resuscitation of patients with severe burns}

\author{
Mette M Berger ${ }^{*}$ and Yok Ai Que \\ See related research by Sánchez-Sánchez et al., http://ccforum.com/content/17/4/R176
}

\begin{abstract}
Over-resuscitation is deleterious in many critically ill conditions, including major burns. For more than

15 years, several strategies to reduce fluid administration in burns during the initial resuscitation phase have been proposed, but no single or simple parameter has shown superiority. Fluid administration guided by invasive hemodynamic parameters usually resulted in over-resuscitation. As reported in the previous issue of Critical Care, Sánchez-Sánchez and colleagues analyzed the performance of a 'permissive hypovolemia' protocol guided by invasive hemodynamic parameters (PiCCO, Pulsion Medical Systems, Munich, Germany) and vital signs in a prospective cohort over a 3-year period. The authors' results confirm that resuscitation can be achieved with below-normal levels of preload but at the price of a fluid administration greater than predicted by the Parkland formula (2 to $4 \mathrm{~mL} / \mathrm{kg}$ per\% burn). The classic approach based on an adapted Parkland equation may still be the simplest until further studies identify the optimal bundle of resuscitation goals.
\end{abstract}

The recently published paper by Sanchez-Sanchez and colleagues [1] is a very interesting and well documented study showing how challenging it is to prevent fluid creep during early resuscitation after major burn trauma. The aim of resuscitation is to restore adequate organ and tissue oxygenation. Under- and over-resuscitation have been associated with complications and poor outcome not only in patients with burns [2] but in other critically ill patients

\footnotetext{
* Correspondence: mette.berger@chuv.ch

Service of Adult Intensive Care Medicine \& Burns, Lausanne University Hospital (CHUV BH-08.612), Rue du Bugnon 46, CH 1011 Lausanne, Switzerland
}

as well [3]. Volume creep in major burns contributes to worsening of burn edema, conversion of superficial to deep burns, and compartment syndromes. Although the use of vital signs - heart rate and mean arterial pressure (MAP) - and urine output may lead to under-resuscitation in burned patients who are critically ill [4], several teams have attempted to optimize fluid resuscitation after major burns [5-7]. Basing fluid delivery on invasive hemodynamic monitoring has repeatedly been shown to cause overresuscitation [8]. Some years ago, Arlati and colleagues [9] introduced the concept of permissive hypovolemia. They showed that fluid administration guided by a hemodynamics-oriented approach throughout the first 24-hour period limited to a urine output of 0.5 to $1 \mathrm{~mL} / \mathrm{kg}$ per hour and a cardiac index of at least $2.2 \mathrm{~L} /$ minute per $\mathrm{m}^{2}$ was safe and resulted in less organ dysfunction [9]. This year, Sánchez-Sánchez and colleagues [1] present interesting results of a 3-year prospective cohort study of 132 consecutive critically ill patients (age of $48 \pm 18$ years) with a burned body surface (total body surface area, or TBSA) of $35 \pm 22 \%$.

The resuscitation algorithm was guided by blood pressure (MAP of more than $65 \mathrm{~mm} \mathrm{Hg}$ ), urinary output ( 0.5 to $1 \mathrm{~mL} / \mathrm{kg}$ ), transpulmonary thermodilution, and lactate levels: the crude $4 \mathrm{~mL} / \mathrm{kg}$ per TBSA formula was used as a starting value at the beginning of the resuscitation but was constantly adjusted to achieve (a) a cardiac index of more than $2.5 \mathrm{~L} /$ minute per $\mathrm{m}^{2}$, (b) an intrathoracic blood volume index (ITBVI) of more than $600 \mathrm{~mL} / \mathrm{m}^{2}$, and (c) normalization of lactate levels by 32 hours. The mean fluid rate required to achieve the protocol targets in the first 8 hours was $4.05 \mathrm{~mL} / \mathrm{kg}$ per TBSA burned, increasing during the next 16 hours. The authors conclude that the initial burn trauma-induced hypovolemia is not optimally reflected by MAP and hourly urine output but can be detected by transpulmonary thermodilution during the early resuscitation phase. The authors consider that, 
thanks to their protocol, unnecessary fluid overload was prevented.

However, there are some concerns with the results presented by Sánchez-Sánchez and colleagues [1] and doubts about the achievement of what they presented as 'dry resuscitation', especially when strong endpoints such as intra-abdominal pressure and Sepsis-related Organ Failure Assessment (SOFA) are considered. First, given a mean TBSA of $35 \%$, the mortality rate is elevated (23\%). Second, $12 \%$ of patients have an abdominal compartment syndrome (intra-abdominal pressure of more than $20 \mathrm{~mm} \mathrm{Hg}$ ), of whom $50 \%$ die. Third, acute renal failure occurred in $31.1 \%$ of patients and $11.4 \%$ finally required renal replacement therapy. Fourth, the length of mechanical ventilation is high (21.5 days), and $24.2 \%$ of patients developed acute respiratory distress syndrome, despite a very low prevalence $(9.1 \%)$ of proven inhalation. The persistence of relatively high SOFA scores on days 5 and 7 differs from the fast organ failure decay observed by Arlati and colleagues [9] with a stricter hypovolemia protocol. Finally, early progressive enteral nutrition (EN) - that is, started within 12 hours of injury - has been shown to improve outcome after major burns and should be integrated in any resuscitation protocol since it enhances gut perfusion in both animals and humans, reduces both pyloric dysfunction [10] and gut edema [11], and hence improves gut function: further early EN is associated with a significant reduction of the requirement for parenteral nutrition (PN). In the Spanish cohort, EN was introduced only 'whenever possible', forcing the use of PN in $61.3 \%$ of patients, which is unusual and is likely to reflect gut dysfunction.

Despite showing the willingness to reduce fluid delivery, the above events might still reflect over-resuscitation. Indeed, the ITBVI-driven resuscitation protocol results in a fluid delivery above the Parkland-determined target, which by no means can be qualified as a 'hypovolemic' target. Remember that the Advanced Trauma Life Support (ATLS) guidelines now recommend considering the low range of 2 to $4 \mathrm{~mL} / \mathrm{kg}$ per\% TBSA of the Parkland formula for calculation of the first 24 hours' fluid administration [12]! Furthermore, the resuscitation algorithm does mention the use of inotropes and vasopressors, but only after attempting changes in fluid load. This may be the missing link to explain higher-than-expected fluid administration. Unfortunately, the results do not give details on the use of the latter or track the gain of weight.

In conclusion, though not achieving the degree of overresuscitation described by Holm and colleagues [8], the strategy by Sánchez-Sánchez and colleagues [1] still suffers from some degree of fluid overload and did not achieve the expected reduction of organ failures (pulmonary, renal, and digestive). Some degree of hypovolemia during the first 24 hours after burn does not require complete correction when occurring under close supervision. The ATLS proposed guidance by the low range of the Parkland formula remains a good alternative to an invasive hemodynamic target to guide initial resuscitation after major burns.

\section{Abbreviations}

ATLS: Advanced Trauma Life Support; EN: Enteral nutrition; ITBVI: Intrathoracic blood volume index; MAP: Mean arterial pressure; PN: Parenteral nutrition; SOFA: Sepsis-related Organ Failure Assessment; TBSA: Total body surface area.

\section{Competing interests}

The authors declare that they have no competing interests.

\section{Published: 11 Nov 2013}

\section{References}

1. Sánchez-Sánchez M, Garcia-de-Lorenzo A, Herrero E, Lopez T, Galvan B, Asensio MJ, Cachafeiro L, Casado C: A protocol for resuscitation of severe burn patients guided by transpulmonary thermodilution and lactate levels: a 3-year prospective cohort study. Crit Care 2013, 17:R176.

2. Pruitt BA Jr: Protection from excessive resuscitation: 'pushing the pendulum back'. J Trauma 2000, 49:567-568.

3. Payen D, de Pont AC, Sakr Y, Spies C, Reinhart K, Vincent JL: A positive fluid balance is associated with a worse outcome in patients with acute renal failure. Crit Care 2008, 12:R74.

4. Dries DJ, Waxman K: Adequate resuscitation of burn patients may not be measured by urine output and vital signs. Crit Care Med 1991, 19:327-329.

5. Klein MB, Hayden D, Elson C, Nathens AB, Gamelli RL, Gibran NS, Herndon DN, Arnoldo B, Silver G, Schoenfeld D, Tompkins RG: The association between fluid administration and outcome following major burn: a multicenter study. Ann Surg 2007, 245:622-628.

6. Saffle JR: The phenomenon of 'fluid creep' in acute burn resuscitation J Burn Care Res 2007, 28:382-395.

7. Tricklebank S: Modern trends in fluid therapy for burns. Burns 2009, 35:757-767.

8. Holm C, Melcer B, Hörbrand F, von Donnersmarck GH, Mühlbauer W: The relationship between oxygen delivery and oxygen consumption during fluid resuscitation of burn-related shock. J Burn Care Rehabil 2000, 21:147-154

9. Arlati S, Storti E, Pradella V, Bucci L, Vitolo A, Pulici M: Decreased fluid volume to reduce organ damage: a new approach to burn shock resuscitation? A preliminary study. Resuscitation 2007, 72:371-378.

10. Raff T, Hartmann B, Germann G: Early intragastric feeding of seriously burned and long-term ventilated patients: a review of 55 patients. Burns 1997, 23:19-25.

11. Rousseau AF, Losser MR, Ichai C, Berger MM: ESPEN endorsed recommendations: nutritional therapy in major burns. Clin Nutr 2013, 32:497-502.

12. American College of Surgeons Committee on Trauma: Thermal injuries. In ATLS: Advanced Trauma Life Support for Doctors (Student Course Manual). 8th edition. Chicago: American College of Surgeons; 2008:211-224.

\section{$10.1186 / \mathrm{cc} 13101$}

Cite this article as: Berger and Que: A protocol guided by transpulmonary thermodilution and lactate levels for resuscitation of patients with severe burns. Critical Care 2013, 17:195 